\title{
Association phenomenon of esters in water/surfactant/co-surfactant/hydrocarbon system
}

\begin{abstract}
The pseudoternary phase diagrams of water/sodium dodecyl suphate(SDS):hexan-1ol(35:65)/hydrocarbon/esters were prepared. The esters used are ethyl-n-butyrate, ethyl benzoate, methyl oleate and methyl linolenate. While the hydrocarbons are n-decane and pxylene. The solubility area of the W/O microemulsion region was found to be largest in ethyln-butyrate followed by ethyl benzoate, methyl oleate and methyl linolenate for all of the systems. No appreciable difference in the W/O microemulsion region was observed as the ndecane was substituted with p-xylene.
\end{abstract}

Keyword: Esters; Solubility 